\title{
A CONCEPÇÃO DOS PROFISSIONAIS ACERCA DO PROJETO TERAPÊUTICO DE CENTROS DE ATENÇÃO PSICOSSOCIAL - CAPS*
}

Luciane Prado Kantorski ${ }^{1}$, Valquiria de Lourdes Machado Bielemann² ${ }^{2}$ Bianca Neme Clasen ${ }^{2}$, Maria Angélica Silveira Padilha ${ }^{3}$, Maria Emilia Nunes Bueno ${ }^{4}$, Rita Maria Heck ${ }^{5}$

\begin{abstract}
RESUMO: Estratégias de intervenção nos Centros de Atenção Psicossocial são planejadas e têm objetivos estabelecidos por meio do projeto terapêutico do serviço. O presente trabalho visa identificar a concepção dos profissionais de um Centro de Atenção Psicossocial da Região Sul do Brasil acerca do projeto terapêutico. Trata-se de estudo descritivo de abordagem qualitativa em que se aplicou entrevistas semiestruturadas a 21 trabalhadores, no período de julho a dezembro de 2006. Os resultados foram analisados em duas unidades temáticas: Projeto Terapêutico com Vistas à Integralidade do Cuidado e Projeto Terapêutico com Vistas à Autonomia. O projeto terapêutico, na concepção dos profissionais, é um importante mecanismo para a reabilitação do portador de sofrimento psíquico, sendo uma possibilidade para o desenvolvimento do cuidado integral, favorecendo a aquisição da autonomia e o restabelecimento da inclusão social. Conclui-se que o projeto terapêutico, operacionalizado através dos planos terapêuticos singulares, é uma referência no planejamento do cuidado integral.
\end{abstract}

PALAVRAS-CHAVE: Saúde mental; Políticas de saúde; Avaliação; Reabilitação; Autonomia pessoal.

\section{CAPS' PROFESSIONALS' CONCEIVING ABOUT ITS THERAPEUTIC PROJECT}

ABSTRACT: Intervention strategies in Psychosocial Care Centers (CAPS) are planned and have established goals through the therapeutic service planning. This study aims to identify the CAPS' professional's conceiving regarding the therapeutic project held in Southern Brazil. It is a descriptive study of qualitative approach in which semi-structured interviews applied to 21 workers during the period July to December 2006 were performed. The results were analyzed in two thematic units: Therapeutic Project and Integrality of Care and Therapeutic Project and Autonomy. The therapeutic plan, accordingly to the professionals' conceiving, is an important mechanism for the rehabilitation of patients with psychological distress, with a possibility for the development of comprehensive care, facilitating the acquisition of autonomy and the restoration of social inclusion. It is concluded that the therapeutic project, operated through unique treatment plans is a reference for the comprehensive care planning.

KEYWORDS: Mental health; Health policy; Evaluation; Rehabilitation; Personal autonomy.

\section{LA CONCEPCIÓN DE LOS PROFESIONALES ACERCA DEL PROYECTO TERAPÉUTICO DE CENTROS DE ATENCIÓN PSICOSOCIAL - CAPS}

RESUMEN: Las estrategias de intervención en los Centros de Atención Psicosocial son planeadas y tienen objetivos establecidos por medio del proyecto terapéutico del servicio. El presente trabajo pretende identificar la concepción de los profesionales de un Centro de Atención Psicosocial de la Región Sur de Brasil acerca del proyecto terapéutico. Se trata de estudio descriptivo de abordaje cualitativo en que se hicieron entrevistas semiestructuradas a 21 trabajadores en periodo de julio a diciembre de 2006. Los resultados fueron analizados en dos unidades temáticas: Proyecto Terapéutico con Vistas a la Integralidad del Cuidado y Proyecto Terapéutico con Vistas a la Autonomía. El proyecto terapéutico, en la concepción de los profesionales es un importante mecanismo para la reabilitación del portador de sufrimiento psíquico, siendo una posibilidad para el desarrollo del cuidado integral, favoreciendo la adquisición de la autonomía y el restablecimiento de la inclusión social. La conclusión es que el proyecto terapéutico, operacionalizado por medio de los planes terapéuticos singulares es una referencia en el planeamiento del cuidado integral.

PALABRAS CLAVE: Salud mental; Políticas de salud; Evaluación; Reabilitación; Autonomía personal.

*Pesquisa financiada pelo Conselho Nacional de Desenvolvimento Científico e Tecnológico (Processo n 554537/2005-2).

${ }^{1}$ Enfermeira. Doutor em Enfermagem. Professor Adjunto da Faculdade de Enfermagem da Universidade Federal de Pelotas-UFPel. Pesquisador do CNPq.

${ }^{2}$ Enfermeira. Mestre em Assistência de Enfermagem. Professora Adjunta da Faculdade de Enfermagem da UFPel.

${ }^{3}$ Enfermeira. Mestranda do Programa de Pós-Graduação em Enfermagem da Faculdade de Enfermagem da UFPel

${ }^{4}$ Enfermeira. Mestranda do Programa de Pós-Graduação em Enfermagem da UFPel. Bolsista de Apoio Técnico CNPq.

${ }^{5}$ Enfermeira. Doutor em Enfermagem. Professor Adjunto da Faculdade de Enfermagem da UFPel.

Autor correspondente:

Luciane Prado Kantorski

Universidade Federal de Pelotas

R. Victor Valpírio, 289 - 96020-250 - Pelotas-RS-Brasil

Recebido: 07/06/10

E-mail:kantorski@uol.com.br

Aprovado: 20/10/10 


\section{INTRODUÇÃO}

As formas de lidar com a doença e com os doentes mentais, no Brasil, nas últimas décadas, vêm sofrendo transformações. Substituindo o modelo manicomial, emergiram movimentos de reforma psiquiátrica em diversos países, inclusive no Brasil. O modelo daqui é uma proposta diferenciada para cuidar dos portadores de sofrimento psíquico, consistindo em um modelo de atenção que se propõe a assistir na comunidade, resgatar a cidadania e promover a reabilitação psicossocial.

Dentro desse foco, o processo de reabilitação psicossocial favorece ao indivíduo exercer a cidadania e adquirir autonomia, formação profissional, capacidade social e para o trabalho, sendo uma possibilidade de desenvolver nessas pessoas a capacidade de exercer plenamente seus direitos, construir a própria habilidade de acesso ao valor, no caminho da emancipação. Busca, ainda, estabelecer melhores negociações entre as necessidades dos portadores de sofrimento psíquico e as oportunidades do contexto ${ }^{(1)}$.

No Brasil, o marco político da Reforma Psiquiátrica respalda-se na Lei Federal 10.216, de 6 de abril de 2001, a qual dispõe sobre a proteção e os direitos das pessoas portadoras de transtornos mentais e redireciona o modelo assistencial em saúde mental $^{(2)}$. A ela se antecede a Lei Estadual 9.716, de 7 de agosto de 1992, que dispõe sobre a reforma psiquiátrica no Rio Grande do Sul. Ressalta-se que este estado brasileiro é pioneiro no país no que se refere à legitimação da política de reforma psiquiátrica, determinando a substituição progressiva dos leitos nos hospitais psiquiátricos por redes de atenção integral em saúde mental e instituindo regras de proteção aos que padecem de sofrimento psíquico, especialmente quanto às internações psiquiátricas compulsórias ${ }^{(2)}$.

A Reforma Psiquiátrica edifica-se na assistência prestada pelos serviços substitutivos que funcionam sob a égide de serviços de saúde mental e das redes sociais. Cabe ressaltar que as descrições de rede estão baseadas nos conceitos de laços, atrelados a um sistema social. Esses laços conectam pessoas, grupos e organizações sociais. No seu conceito sociológico, a rede destaca a vida cotidiana como espaço estratégico para articulação política e institucional das comunidades e grupos sociais ${ }^{(3)}$.

Os Centros de Atenção Psicossocial (CAPS), serviços estratégicos na assistência ao sofredor psíquico no contexto social e de saúde, são responsáveis pela organização da demanda e da rede de cuidados em saúde mental, no âmbito de seu território. Esses serviços normatizam-se a partir da Portaria GM/336, de 19 de fevereiro de $2002^{(2)}$.

Nos CAPS, as estratégias de intervenção são planejadas e têm objetivos estabelecidos através do projeto terapêutico que, de acordo com a Portaria 147, de 25 de agosto de 1994, defini-se como o conjunto de objetivos e ações estabelecidos e executados pela equipe multiprofissional, voltados para a recuperação do usuário do serviço, desde a admissão até a alta. É um instrumento de gestão que deve contemplar o desenvolvimento de programas específicos e interdisciplinares adequados às características da clientela, compatibilizando a proposta de tratamento com a necessidade de cada usuário e de sua família( ${ }^{(2)}$.

O projeto terapêutico do serviço deve compor modelos assistenciais que rompam com as formas excludentes de tratamento ao portador de distúrbio mental. Portanto, esse projeto precisa ir na direção da reabilitação psicossocial, proporcionando o desenvolvimento do sujeito como ator principal de sua vida e de seu tratamento, com direitos sociais. Desta forma, o CAPS precisa ter o compromisso de inverter o modelo manicomial, asilar e excludente, e funcionar como um mediador de ações que possibilitem a reabilitação e inserção social dos sujeitos ${ }^{(4)}$.

Entende-se que o projeto terapêutico é uma ferramenta importante para o desenvolvimento do cuidado ao portador de sofrimento psíquico, pois serve de eixo de referência para guiar as ações dos profissionais dos serviços substitutivos na busca de atender à reabilitação psicossocial do sofredor psíquico. Sendo assim, o presente artigo tem como objetivo identificar a concepção dos profissionais de um CAPS acerca do projeto terapêutico do serviço no qual atuam.

\section{METODOLOGIA}

Esse estudo é um recorte da Pesquisa "Avaliação dos Centros de Atenção Psicossocial-CAPSUL" da Região Sul do Brasil (Rio Grande do Sul, Santa Catarina e Paraná), que se desenvolveu em duas etapas, uma quantitativa e outra qualitativa. A etapa qualitativa concentrou-se em CAPS I e II, de cinco municípios da Região Sul do país. São eles: Alegrete, Joinville, São Lourenço do Sul, Porto Alegre e Foz do Iguaçu. As avaliações foram desenvolvidas na forma de cinco estudos de caso, e este artigo se dedica a analisar dados de um dos estudos, referente a um dos municípios. Caracteriza-se como um estudo 
descritivo, de abordagem qualitativa ${ }^{(5)}$.

O local da pesquisa foi o CAPS Nossa Casa e sua realização ocorreu no período de julho a dezembro de 2006. Esse serviço funciona como espaço de cuidado em saúde mental desde 1988; atende pessoas do meio urbano e rural e serve como referência aos municípios mais próximos. Atua com uma equipe multiprofissional composta por 23 trabalhadores ${ }^{(5)}$.

O município em questão pode ser considerado um referencial no país na assistência substitutiva aos hospitais psiquiátricos. Apresenta uma particularidade importante frente a outros municípios, que foi a criação de um Centro Comunitário de Saúde Mental, mesmo antes da regulamentação das Leis Estadual e Federal ${ }^{(5)}$.

Os sujeitos do estudo foram 21, do total de 23 trabalhadores, em virtude de duas recusas. Os critérios de inclusão foram: ter vínculo empregatício com o serviço, aceitar participar do estudo através da assinatura do Termo de Consentimento Livre e Informado, e a representatividade multiprofissional - ao menos um sujeito de cada profissão ${ }^{(5)}$.

A pesquisa respeitou os preceitos éticos vigentes nas Resoluções n. 196/96 e n. 251/97 do Ministério da Saúde/Conselho Nacional de Saúde do Brasi $^{(6)}$ e se desenvolveu após a aprovação do Comitê de Ética em Pesquisa da Faculdade de Medicina da Universidade Federal de Pelotas, mediante Ofício $n^{\circ}$ 074/05, de 11 de novembro de 2005.

Os dados foram obtidos por meio de entrevistas semiestruturadas, utilizando-se da seguinte questão norteadora: Como o projeto terapêutico orienta o trabalho do CAPS? As entrevistas foram gravadas, transcritas na íntegra, lidas exaustivamente, analisadas e organizadas em duas unidades temáticas, a saber: Projeto Terapêutico com Vistas à Integralidade do Cuidado e Projeto Terapêutico com Vistas à Autonomia. Os fragmentos de fala presentes neste estudo foram identificados através da letra "E", para os profissionais da equipe, e acompanhados de numeração que corresponde à ordem das entrevistas realizadas.

\section{RESULTADOS E DISCUSSÃO}

Observa-se que os profissionais de saúde do CAPS destacaram dois pontos importantes que devem integrar o projeto terapêutico, à medida que corroboram com o processo de construção da reabilitação psicossocial, a qual busca para o portador de sofrimento psíquico a construção de uma nova forma de vida, autônoma e inserida na sociedade.
Sendo assim, apresenta-se e discute-se a primeira unidade temática: Projeto terapêutico com vistas à integralidade do cuidado.

O cuidado integral pode ser compreendido como um conjunto de noções pertinentes a uma assistência livre de reducionismo. Há, nessa concepção, uma visão abrangente do ser humano, o qual é dotado de sentimentos, desejos e aflições, ou seja, considera-se a subjetividade como o fenômeno que se sobressai ${ }^{(7)}$. Esse tipo de atendimento pode contribuir muito para garantir a qualidade da atenção à saúde e, consequentemente, $o$ avanço da qualidade de vida do usuário.

A fim de buscar a qualidade na assistência dentro dos CAPS, o projeto terapêutico precisa voltarse para as necessidades individuais dos usuários, devendo, portanto, ser flexível, sofrendo sistematicamente a avaliação da equipe, visando um atendimento de excelência ${ }^{(8)}$. Essa consideração pode ser evidenciada nas falas abaixo, nas quais está presente a preocupação com relação à adequabilidade do projeto terapêutico do serviço às necessidades e habilidades dos usuários.

O projeto terapêtico tem que estar envolvido na integralidade. Ele tem que estar envolvido em tudo o que nós vamos fazer, quando chegar ao tratamento, à reabilitação. Ele [falando do usuário] não pode ir para um hospital psiquiátrico, o dispositivo de internação é o hospital geral. [...] apresenta características diferenciais, aonde a família faz parte do processo. (E 12)

Eu fiz esse grupo de mulheres, pois notei que o que elas queriam era uma ensinar à outra alguma coisa que sabiam que era terapêutico. Uma sabia tricô, outra sabia fazer sacola pet [...] foi um espaço que eu criei; elas têm ficado alguns minutos a mais para ensinar para a outra, pelo espaço terapêutico. Aí, vão se formando esses espaços, isso é importante. (E 10)

Nesses relatos sobressai que a proposta contida no projeto terapêutico deve contemplar a integralidade do cuidado ao usuário, estabelecendo e fortalecendo os vínculos familiares. O serviço precisa estar próximo à família e inserido no processo de reabilitação.

Além disso, para dar conta desta fundamentação prevista na Reforma Psiquiátrica, a referência da internação passa a ser o hospital geral, na tentativa de desmistificar a loucura e respeitar a singularidade do ser, com suas diferenças. Cabe salientar que, na vigência da internação, a equipe do CAPS se mantém imbuída da responsabilidade da manutenção do vín- 
culo com o usuário e grupo familiar, estabelecendo o acompanhamento e fortalecendo a rede de conversação, que prevê a manutenção de diálogos e da responsabilização dos profissionais pelos usuários.

Frente a essa discussão, é preciso considerar que a integralidade do cuidado ao usuário deve ser prioritária, percebendo o indivíduo como sujeito histórico, social e político, articulado ao seu contexto familiar, bem como ao meio ambiente e à sociedade na qual se insere ${ }^{(9)}$. Sendo assim, a importância de desenvolver a assistência de forma integral está fundamentada na articulação de todas as formas da produção do cuidado.

Destaca-se, ainda, que o cuidado integral deve compreender ações preventivas, curativas e de promoção de saúde no âmbito individual e coletivo, e nos diferentes níveis de complexidade ${ }^{(10)}$. O que se segue são colocações que corroboram com o autor.

[...] o usuário como alguém que precisa de um cuidado, e os equipamentos que nós temos são: a interrelação no projeto terapêutico, todos os recursos que o território nos traz, a integralidade da ação que trabalha promoção, prevenção, tratamento e reabilitação; trabalhar de uma forma integral. (E 12)

Nesse sentido, para que o restabelecimento da saúde mental seja efetivo, é necessário ter um olhar para as diversas formas de abordagem do cuidado, trabalhando a reabilitação psicossocial, através da promoção da saúde e prevenção de enfermidades de maneira integral.

Considerando que a equipe de trabalhadores do CAPS inclui diferentes profissões e saberes, colocase em evidência a necessidade da interdisciplinaridade como importante ferramenta a ser utilizada dentre os diversos recursos dos serviços substitutivos, na vertente da terapêutica da integralidade do cuidado. A interdisciplinaridade é entendida como a interação de vários saberes sobre um mesmo objeto. Nessa perspectiva, existe a intercomunicação entre as disciplinas, de forma que resulte numa troca entre elas, através do diálogo operante ${ }^{(11)}$.

O cuidado integral vem sendo um desafio na construção de novos modelos assistenciais. A inserção social de pessoas vulneráveis e o desenvolvimento de formas de convívio com a diferença exigem transformações profundas nos modos de conceber o cuidado e organizar os serviços, em confronto com as concepções e estratégias tradicionais, o que implica na definição de novos perfis profissionais ${ }^{(12)}$.

Acredita-se que as práticas calcadas no novo modelo de intervenção dêem conta de cuidar na perspectiva da integralidade. Assim, pensa-se ser possível favorecer o processo de reinserção social do sujeito com transtorno psíquico, contribuindo para sua reabilitação. As falas a seguir mencionam esta consideração.

O tratamento aqui não se resume só a um atendimento médico, têm atividades que são propostas, atividades que esse serviço tem desde o início. A cada momento de crescimento, de demanda, de complexidade social e de complexidade contemporânea, se colocam outras coisas dentro desse projeto terapêutico, e essas coisas geralmente surgem exatamente pela necessidade. (E 2)

Esse projeto tem como objetivos a melhora do usuário e a reinserção de cada um. O projeto terapêutico é bem para isso, [...] recuperar as coisas que perdeu, ou pelo menos fazer uma troca, [...] tentar recuperar uma vida que às vezes fica meio estacionada. (E 8)

O que vejo é uma mudança, principalmente nos usuários que chegam. Eles chegam com os seus medos, as suas ansiedades. Em questão de uma semana, a gente já vê perfeitamente a diferença neles. Eles já se sentem mais seguros, mais aceitos, eles têm um convivio melhor nesse sentido. (E 13)

Nos depoimentos fica explícito que o modelo substitutivo de atenção à saúde mental contempla uma perspectiva interdisciplinar com vistas à integralidade do cuidado, em detrimento do modelo tradicional, que é centrado na figura do médico. Além disso, está presente a flexibilidade do serviço, que incorpora novos modos de fazer saúde mental, em resposta às complexas demandas da sociedade contemporânea.

Nessa ótica, acredita-se que os serviços substitutivos podem representar espaços de ações que vão em direção ao discurso apresentado pelos depoentes, na medida em que consistem em locais de atenção diária, abertos, de livre acesso aos usuários, familiares e sociedade. Os serviços comunitários devem contar com equipe multiprofissional, voltada ao atendimento das demandas integrais aos indivíduos e suas famílias ${ }^{(13)}$. Além disso, transparece que a proposta do serviço contida no projeto terapêutico pode apresentar uma perspectiva abrangente diante da reabilitação do portador de sofrimento psíquico. 
Para tanto, a equipe precisa voltar suas ações para a construção, junto ao usuário, do projeto terapêutico singular, que deve prever o atendimento das suas necessidades e possibilitar o desenvolvimento do seu potencial, capacitando-o para um viver melhor, portanto, qualificando sua vida.

Coerentemente, surge também a necessidade da atenção básica de saúde constituir, de fato, junto aos demais serviços de saúde mental, a rede articulada e integrada à proposta da Reforma Psiquiátrica, no intuito de contemplar a integralidade do cuidado. Sendo assim, é imprescindível que tenha, para com os outros serviços que constituem a rede de saúde mental, a concepção da corresponsabilização frente às demandas de promoção, manutenção e reabilitação do portador de sofrimento psíquico do seu território.

Nas colocações que se seguem, evidencia-se que existe uma proposta de cuidado integral que resgata as potencialidade do usuário para desenvolvê-lo como pessoa com direitos e deveres.

Existe um caminhar do serviço e o caminhar dos usuários: o que nós queremos, onde queremos chegar, o que ele precisa, aonde ele precisa de mais auxílio, qual a sua potencialidade, o que ele pode fazer para ser mais feliz; no que pode se ocupar para ter mais, para estar mais de acordo com o seu talento. (E 3)

Então, a promoção em saúde mental vai ser otimizada quando a rede básica de saúde estiver, também, cada vez mais ligada à vida das populações. (E 12)

Neste contexto, vislumbram-se novas práticas de atuação em saúde mental, resgatando a necessidade da rede básica firmar laços com a rede de saúde mental propriamente dita, o que contribui na promoção da assistência, através do intercâmbio de ações de saúde, e pode colaborar para desmistificar a doença e facilitar a ressocialização do usuário.

A magnitude da atenção integral em saúde mental se edifica na medida em que todos os componentes das ações terapêuticas estão voltados a uma lógica comum, que busca evitar a fragmentação dos sujeitos, das necessidades e das ações. Assim, a construção da integralidade configura não só um campo prático diferenciado, mas também uma nova ética do cuidado $^{(11)}$.

Outro aspecto importante do processo de reabilitação do usuário do serviço de saúde mental, e ligado ao projeto terapêutico, é a autonomia, para a qual dá-se ênfase na segunda unidade temática abordada: Projeto terapêutico com vistas à autonomia.

A autonomia do ser humano está associada à sua independência, ou seja, à sua capacidade de tomar decisões a respeito de si próprio, tanto com relação à sua saúde, como também à integridade físico-psíquica e relações sociais ${ }^{(14)}$. Entende-se que a autonomia está relacionada a condições de saúde favoráveis, possibilitando ao indivíduo a capacidade de se autogovernar, de obter direitos de liberdade, privacidade e escolha dos próprios comportamentos.

O projeto terapêutico do serviço, assim como os planos terapêuticos singulares, precisam ser pensados e construídos de forma a contemplarem aspectos de empoderamento relacional e social, o que significa o aumento do poder e autonomia pessoal e coletiva de indivíduos e grupos sociais, nas relações interpessoais e institucionais, principalmente daqueles submetidos às relações de opressão, discriminação e dominação social $^{(15)}$.

Pode-se evidenciar, nas falas a seguir, que a adesão dos usuários às atividades possibilita o desenvolvimento de habilidades laborativas, o que visa trabalhar justamente as capacidades de independência, liberdade e autonomia.

Quando a gente faz as oficinas, a gente tem o intuito de fazer uma coisa que eles aprendam e aprendam a fazer bem. (E 6)

O tapete, logicamente, é uma coisa ótima. De repente, eles melhoram, têm alta, eles começam a fazer um trabalho para eles mesmos, já têm uma renda [...]. E aquilo eles aprendem, para eles é ótimo. Acho que todas as oficinas foram pensadas para isso, para que eles tenham futuramente um desenvolvimento para eles próprios. (E 7)

Ele [coordenador] criou a carrocinha de vender cachorro-quente, ela [usuária] sai nas ruas para vender cachorro-quente, os meninos saem para vender picolé e tudo. Ele [coordenador] acha que aqui eles têm capacidade de fazer essas coisas. (E 4)

Isso até se aproveita para, futuramente, em pessoas que possam até adquirir um emprego através disso. Se eles trabalham bem na cozinha, futuramente podem trabalhar em um restaurante. Foi a maneira de pensar que as pessoas podem melhorar suas vidas pessoais e suas casas. (E 19) 
[...] oficinas profissionalizantes envolvidas também nesse contexto da economia solidária que hoje nós estamos trabalhando [...]; o projeto de geração de renda é bem legal, vai gerar um retorno, assim, bem legal de trabalho. (E 21)

A independência financeira é outra questão citada pelos entrevistados. Conquistá-la pode ser considerado um fator importante para a autonomia e reabilitação do usuário. $\mathrm{O}$ trabalho é uma forma de materializar um lugar de integração social, além de ser reconhecido como um meio de reproduzir a vida material dos sujeitos ${ }^{(16)}$.

Apostar nestas pessoas consiste em acreditar na potencialidade dos indivíduos com transtorno psíquico para a mudança de padrões e comportamentos, e para a aquisição de habilidades que lhes permitam viver e interagir em sociedade. Esse viver pode dar-se através da inserção real no mercado de trabalho, como também pelo mínimo gerenciamento - o cuidado consigo mesmo, com o seu próprio tratamento, do ambiente, das suas relações interpessoais, do que for capaz $^{(8)}$.

A seguir, os entrevistados colocam, também, a questão da autonomia como um dos objetivos principais do projeto terapêutico, tentando resgatar em cada usuário a própria habilidade de fazer com que ele utilize-se disso para seu próprio benefício e independência.

Eu acho que a construção do plano terapêutico singular, juntamente com o usuário, é uma das coisas mais importantes que eu vejo. E até tem que ser colocado isso como uma coisa dentro do projeto terapêutico, como uma coisa principal. (E 1)

O nosso maior objetivo, quanto ao projeto terapêtico, é fazer essa inclusão na comunidade, ter na Nossa Casa um acompanhamento de orientação, de tentar buscar as habilidades e as dificuldades que eles têm, para que possam exercer na sua rotina diária, mas que eles possam sair, na medida do possivel, dando aula. (E 18)

O plano terapêutico singular é uma pactuação entre o serviço e o usuário. Deve ir ao encontro das necessidades e potencialidades dos sujeitos em sofrimento psíquico. Embora se consolide a partir da proposta do projeto terapêutico do serviço, ambos devem entrelaçar-se para corresponder às demandas de saúde do usuário. É importante salientar que investir nas potencialidades dos sujeitos, em que a subjetividade de cada ser humano seja um aspecto a ser considerado, é um caminho a desenvolver autonomia, possibilitando crescimento pessoal e respeito a si próprio ${ }^{(17)}$.

Para que exista esse investimento, é necessário que se ofereça espaço ao usuário para desenvolver potencialidades, através das atividades dentro do projeto terapêutico do serviço, bem como junto a seus familiares, praticando atividades do cotidiano com vistas à inclusão social, o que se pode evidenciar nas falas abaixo:

A gente procura produzir a inclusão mesmo. O projeto terapêutico tem de trabalhar nessas dificuldades, trazer os familiares para que eles multipliquem dentro de suas casas e consigam trabalhar isso, e não achem que eles [usuários] não podem cortar uma carne, usar uma faca. Nós temos facas, garfos, temos tudo e eles usam. Eles não comem com colher. Em um restaurante não vão comer com colher. Então, eles usam, podem usar. Tem tesouras para cortarem papel, pintarem. A gente não separa eles, no dia a dia, das tarefas diárias. A gente coloca eles dentro, para saberem como vão lidar com aquilo. (E 18)

Eles se sentam tudo ali e ficam fazendo grupo, lendo jornal. Dão uma conversada. Estão sempre meio por dentro do que acontece. Hoje, eles já estão falando em eleição; quando eu saí, eles já estavam falando das eleições de domingo. (E 4)

A gente não pode criar uma oficina sem que essa oficina beneficie o usuário. Ele é o principal interessado. Então, dentro disso que a gente cria. Quando a gente está se apresentando [coral e dança] [...]. E se não fosse nós, Nossa Casa, proporcionar isso a eles, eles jamais iam sair [...], eles jamais iam conhecer outra cidade. Isso eu acho muito importante. (E 15)

As atividades domésticas promovem o ajustamento do homem ao social, transformam o cotidiano em ações que orientam o fazer prático do ser humano, como legitimador da ordem social ${ }^{(18)}$. Ser capaz de cuidar da casa é um dos primeiros passos para se conquistar independência e viver na sociedade, sem precisar do auxílio diário de outras pessoas ${ }^{(1)}$.

Pode-se pensar em autonomia como o momento em que o sujeito passa a conviver com seus problemas de forma a requerer menos dispositivos assistenciais do próprio serviço. Desse modo, caberia 
ao CAPS funcionar como um espaço intermediário, um local de passagem, na medida em que possibilita aos usuários um melhor desempenho de suas atividades e autonomia, favorecendo a reinserção social. Tal constatação verifica-se nas declarações que se seguem, quando se referencia o serviço do CAPS como um local de passagem para a conquista de um novo espaço dentro da sociedade.

A gente não quer que ele permaneça só aqui. Que o CAPS seja o espaço para ele conquistar o espaço na rua, dentro da comunidade, dentro da sua casa. Então, a gente procura inclui-lo sempre em tudo que é atividade que tem fora. Fazer com que eles participem das atividades de organização de dentro da casa. $O$ objetivo é criar espaços para que aprendam e consigam ter uma independência dentro das suas casas, da sua comunidade, da sua cidade. (E 18)

Com base no exposto, percebe-se que a compreensão que esse trabalhador tem sobre autonomia vai ao encontro dos preceitos da Reforma Psiquiátrica, de maneira que o mesmo expressa um olhar ampliado em relação ao sujeito acometido de transtorno psíquico.

Os depoimentos identificam que o portador de sofrimento psíquico precisa de ajuda através de múltiplos fazeres, para possibilitar autonomia visando a ressocialização. Além disso, os relatos traduzem a compreensão ampliada dos trabalhadores sobre a importância das ações sustentadas nos fenômenos da subjetividade e da singularidade dos usuários do serviço.

Não há dúvida de que espaços como o CAPS e demais serviços substitutivos permitem que os sujeitos em sofrimento psíquico e as implicações decorrentes desses sofrimentos passem a tomar outra dimensão e sejam considerados sob outro ponto de vista pela sociedade e pelos profissionais de saúde ${ }^{(19)}$.

\section{CONSIDERAÇÕES FINAIS}

Neste estudo, identificou-se que a grande maioria dos entrevistados é conhecedora dos objetivos do projeto terapêutico, da sua importância e significado para a Reforma Psiquiátrica, e que o conhecimento acerca do tema e de suas nuances é um preditor da assistência qualificada, além de ser fundamental para a consolidação do modelo comunitário de atenção em saúde mental.

Aponta-se como limite deste estudo a neces- sidade de aprofundamento entre as concepções sobre o projeto terapêutico, o contexto da Reforma Psiquiátrica e os conflitos enfrentados cotidianamente nos serviços com vistas a superar as práticas manicomiais. Entende-se que outros estudos poderão contribuir no sentido de analisar concepções e práticas como partes de um mesmo cenário em construção.

As declarações expressas nas entrevistas denotam o envolvimento e o comprometimento dos trabalhadores com relação às concepções e práticas reformistas, o que parece imprescindível para a sustentação e consolidação do modelo.

Entender os sujeitos nas suas demandas singulares é fundamental e suscita um cuidado com base na construção minuciosa e pormenorizada do plano terapêutico singular, sedimentado no projeto terapêutico do serviço. Perceber o projeto terapêutico do CAPS como um instrumento indispensável e potencializador do processo de reabilitação psicossocial é concebêlo de forma a sustentar aspectos de empoderamento, autonomia e integralidade.

\section{REFERENCIAS}

1. Salles MM, Barros S. Vida cotidiana após adoecimento mental: desafio para atenção em saúde mental. Acta Paul Enferm. 2009; 22(1):11-6.

2. Ministério da Saúde (BR). Secretaria-Executiva. Secretaria de Atenção à Saúde. Legislação em Saúde Mental:1990-2004. Brasília: Ministério da Saúde, 2004.

3. Martins PH, Fontes B. Redes sociais e saúde: novas possibilidades teóricas. Recife: Editora Universitária da UFPEL; 2008.

4. Milhomem MAG, Oliveira CAGB . O trabalho em equipe nos Centros de Atenção Psicossocial - CAPS. Cogitare Enferm. 2007;12(1):101-8

5. Universidade Federal de Pelotas. Avaliação dos CAPS da Região Sul do Brasil (CAPSUL). Brasília: Conselho Nacional de Desenvolvimento Científico e Tecnológico - CNPq; Ministério da Saúde; 2007. 437p. Relatório final.

6. Ministério da Saúde.(BR). Conselho Nacional de Saúde. Diretrizes e normas regulamentadoras de pesquisas envolvendo seres humanos. Resolução n. 196, de 10 de outubro de 1996. Brasília; 1996.

7. Pinho IC, Siqueita JCBA, Pinho LMO. As percepções 
do enfermeiro acerca da integralidade da assistência. Rev Eletron Enferm. [Internet] 2006; 8(1):42-51 [acesso em 14 set 2009]. Disponível: http://www.fen.ufg.br/ revista/revista8_1/original_05.htm

8. Hirdes A. Autonomia e cidadania na reabilitação psicossocial: uma reflexão. Ciênc Saúde Colet. 2009;14(1):165-71.

9. Machado MFAS, Queiroz DT, Vieira NFC, Barroso, MGT. Integralidade, formação de saúde, educação em saúde e as propostas do SUS - uma revisão conceitual. Ciênc Saúde Colet. [Internet]. 2007 12(2) [acesso em 21 set 2009]. Disponível: http://www.scielo.br/pdf/csc/ v12n2/a09v12n2.pdf

10. Kantorski LP, Souza J, Willrich JQ, Mielke FB. O cuidado em saúde mental: um olhar a partir de documentos e da observação participante. Rev Enferm UERJ. 2006;14(3):366-71.

11. Alves RF, Brasileiro MCE, Brito SMO. Interdisciplinaridade: um conceito em construção. Episteme. 2004;(19):139-48.

12. Mângia EF, Muramoto M. Integralidade e construção de novas profissionalidades no contexto dos serviços substitutivos de saúde mental. Rev Ter Ocup. 2006;17(3):115-22.

13. Mielke FB, Kantorski LP, Jardim VMR, Olschowsky A. Avaliação de um serviço substitutivo em saúde mental. Cogitare Enferm. 2009;14(1):52-8.

14. Arakén AA, Brito AM, Moacir N. Saúde e autonomia: novos conceitos são necessários? Rev Bioét. 2008;16(1):117-24.

15. Vasconcelos EM. O poder que brota da dor e da opressão: empowerment, sua história, teorias e estratégias. São Paulo: EP; 2003.

16. Aranha e Silva AL. O projeto copiadora do CAPS: do trabalho de reproduzir coisas à produção de vida [dissertação]. São Paulo(SP): Universidade de São Paulo; 1997.

17. Francisco BR. Terapia ocupacional. Campinas: Papirus; 2001.

18. Saraceno B. Libertando identidades: da reabilitação psicossocial à cidadania possível. Rio de Janeiro: Instituto Franco Basaglia/Te Cora; 1999.

19. Santos NS, Almeida PF, Venâncio AT, Delgado PG.
A autonomia do sujeito psicótico no contexto da reforma psiquiátrica brasileira. Psicologia Ciência e Profissão [Internet] 2000 20(4) [acesso em 3 out 2009]. Disponível: http://tiny.cc/v47lw 